\title{
CONTROLE DE Acromyrmex laticeps nigrosetosus (HYMENOPTERA: FORMICIDAE), EM EUCALIPTAL NO PARÁ, COM ISCAS GRANULADAS COM SULFLURAMIDA OU CLORPIRIFÓS
}

\author{
José Cola ZANUNCIO', Adalton Pinheiro da CRUZ², Harley Nonato de \\ OLIVEIRA ${ }^{1}$, Francisco Sérgio GOMES ${ }^{2}$
}

RESUMO - Este trabalho foi realizado em reflorestamento de eucalipto da Jari Celulose S.A., no município de Almerim, Pará, de setembro de 1996 a janeiro de 1997. Objetivou-se testar a eficiência de duas iscas granuladas à base de sulfluramida e outra à base de clorpirifós, nas dosagens de 4, 6, 8 e 10 gramas por metro quadrado de formigueiro, para o controle de Acromyrmex laticeps nigrosetosus Forel (Hymenoptera: Formicidae). As duas iscas com sulfluramida, nas dosagens testadas, apresentaram acima de $99,0 \%$ de transporte e nenhuma devolução. Todos os formigueiros tratados, com essas iscas, estavam mortos na avaliação final, enquanto a eficiência da isca com clorpirifós variou de 55,56 a $66,67 \%$, para as diferentes dosagens testadas. As iscas à base de sulfluramida podem ser recomendadas para o controle de A. laticeps nigrosetosus na região do trópico úmido do Brasil.

Palavras-chave: formigas cortadeiras, Eucalyptus, iscas granuladas, Acromyrmex.

Control of Acromyrmex laticeps nigrosetosus (Hym.: Formicidae), in an Eucalyptus Plantation in the State of Pará, Brazil with Granulated Baits with Sulfluramid or Chlorpirifos

\begin{abstract}
This research was developed in an Eucalyprus plantation of Jari Celulose S.A., in Almerin, Pará State, Brazil from September 1996 to January 1997. The objectives were to test the efficiency of two ant baits with sulfluramid and another one with chlorpirifos (at 4, 6, 8 and 10 grams per square meter of ant nest) against Acromyrmex laticeps nigrosetosus Forel (Hymenoptera: Formicidae). Both baits with sulfluramid, in all dosages tested, showed more than $99.0 \%$ of transport with no devolution. All ant colonies treated with these baits were dead during the final evaluation while mortality of nests of this ant treated with chlorpirifos ranged from 55.56 to $66.67 \%$ for the different dosages tested. The sulfluramid baits can be recomended to control A. laticeps nigrosetosus in the humid tropic of Brazil.
\end{abstract}

Key-words: leaf-cutting ants, Eucalyptus, ant baits, Acromyrmex.

\section{INTRODUÇÃO}

As formigas cortadeiras dos gêneros Atta (saúvas) e Acromyrmex (quenquéns) se destacam por danos acentuados aos plantios florestais, especialmente aqueles de eucalipto. As espécies deste último gênero, mesmo apresentando ninhos menores, são um problema sério para o setor florestal, pelo grande número de formigueiros por área (Zanuncio et al., 1996a). Dentre as diferentes espécies de
Acromyrmex, Acromyrmex laticeps nigrosetosus Forel (Hymenoptera: Formicidae) pode ser encontrada nos estados de SP, AM, PA, MA, MG, MT, $\mathrm{GO}, \mathrm{RO}, \mathrm{BA}$ e SC, sendo conhecida vulgarmente como quenquém-campeira (Della Lucia et al. 1993). Seus ninhos são subterrâneos, com um monte de terra saliente sobre a panela ou próximo desta. O substrato para o fungo é retirado de dicotiledôneas. Esta subespécie difere da forma tipica, principalmente pela

\footnotetext{
'Universidade Federal de Viçosa, Departamento de Biologia Animal. Viçosa, MG 36571-000, email: zanuncio@mail.ufv.br

${ }^{2}$ Jari Celulose S.A. Almerim, 68240-000, Monte Dourado, Pará.
} 
coloração castanha-avermelhada da cabeça e do tronco (Gonçalves, 1961).

As formigas cortadeiras forrageiam vegetais, causando prejuízos elevados e competindo com o homem e os animais domésticos (Amante, 1972), além de apresentar grande preferência para a utilização de plantas exóticas (Cherrett, 1968). Assim, o eucalipto como planta exótica cultivada em nosso pais, apresenta muitas espécies preferidas por esses insetos, os quais causam grandes danos aos plantios dessa essência (Mariconi, 1970). Em áreas reflorestadas, principalmente com eucalipto, o controle de formigas deve ser periódico, podendo representar mais de $75,00 \%$ do custo e do tempo total gasto para o controle de pragas nessas áreas (Vilela, 1986).

Neste trabalho, objetivou-se testar a eficiência de duas iscas granuladas à base de sulfluramida e uma à base de clorpirifós, em diferentes dosagens, para o controle da formiga cortadeira $A$. laticeps nigrosetosus.

\section{MATERIAL E MÉTODOS}

Este trabalho foi desenvolvido em plantios de eucalipto da Jari Celulose S.A., no municipio de Almerim, Pará, de setembro de 1996 a janeiro de 1997. A área do ensaio está localizada à latitude $0^{\circ} 54^{\prime} 29^{\prime \prime} \mathrm{S}$, longitude $52^{\circ} 21^{\prime} 50^{\prime \prime} \mathrm{W}$ e altitude de 158 metros. O clima desta região é quente e úmido do tipo Amw, com chuvas concentradas de janeiro a julho e precipitação anual de $2115,0 \mathrm{~mm}$. Na época da instalação deste experimento, o plantio de eucalipto tinha dois anos de idade e altura média de 14 metros. Os tratamentos consistiram na aplicação de três iscas granuladas (duas com sulfluramida e uma com clorpirifós), em diferentes dosagens por metro quadrado de formigueiro (Tab.1), em 18 repetições, sendo cada parcela constituida por um ninho de $A$. laticeps nigrosetosus.

Cada isca granulada nos diferentes tratamentos, foi colocada ao redor dos olheiros ativos dos ninhos de A. laticeps nigrosetosus, avaliando-se o carregamento e ou devolução das mesmas (\% de iscas colocadas que foram carregadas ou devolvidas), 24 horas após sua aplicação. A atividade dos formigueiros foi observada após 3,30 , 50,90 e 120 dias da instalação do ensaio. Nesta última avaliação, os formigueiros foram abertos com enxada para se avaliar a mortalidade dos mesmos, uma vez que esses formigueiros são rasos e não necessitam de sonda para esta avaliação.

\section{RESULTADOS E DISCUSSÃO}

De modo geral, o transporte das iscas mostrou valores semelhantes, em todas as dosagens utilizadas (Tab. 2), demonstrando boa aceitação das mesmas pelas operárias de $A$. laticeps nigrosetosus, sem qualquer devolução.

As iscas granuladas devem ser bem distribuidas nos formigueiros de A. laticeps nigrosetosus que, mesmo pequenos, com tamanho médio de 0,68 a $1,62 \mathrm{~m}^{2}$ apresentam boa atividade, com 3,57 a 4,53 olheiros ativos por metro quadrado de formigueiro (Tab. 1).

Os formigueiros de $A$. laticeps 
Tabela 1. Tratamentos utilizados para o controle de Acrromyrmex laticeps nigrrosetosus $(\mathrm{Hy}-$ menoptera: Formicidae), em Almerim, Pará.

\begin{tabular}{|c|c|c|c|c|c|}
\hline Tratamento & Isca Granulada & $\begin{array}{c}\text { Dosagem } \\
\left(\mathrm{g} / \mathrm{m}^{2}\right)\end{array}$ & $\begin{array}{c}\text { Área Média } \\
\text { dos } \\
\text { Formigueiros } \\
\left(\mathrm{m}^{2}\right)\end{array}$ & $\begin{array}{c}\text { Número Médio } \\
\text { de Canais por } \\
\text { Formigueiro }\end{array}$ & $\begin{array}{l}\text { Número Médio } \\
\text { de Canais por } \mathrm{m}^{2}\end{array}$ \\
\hline 1 & Sulfluramida A $0,3 \%$ & 4 & 0.68 & 2,72 & 4,00 \\
\hline 2 & Sulfluramida A $0,3 \%$ & 6 & 0,69 & 3,11 & 4,51 \\
\hline 3 & Sulfluramida $A \quad 0,3 \%$ & 8 & 0,87 & 3,94 & 4.53 \\
\hline 4 & Sulfiluramida $A \quad 0,3 \%$ & 10 & 1,02 & 3,83 & 3,75 \\
\hline 5 & Sulfluramida B $0,3 \%$ & 4 & 1,62 & 5,78 & 3,57 \\
\hline 6 & Sulfluramida B $0,3 \%$ & 6 & 1,32 & 5,22 & 3,95 \\
\hline 7 & Sulfluramida B $0.3 \%$ & 8 & 1,35 & 4,83 & 3,58 \\
\hline 8 & Sulfuramida B $0,3 \%$ & 10 & 1,33 & 4,89 & 3,68 \\
\hline 9 & Clorpirifos $0,4 \%$ & 4 & 1,01 & 4,17 & 4,13 \\
\hline 10 & Clorpirifós $0,4 \%$ & 6 & 1,07 & 3,89 & 3,64 \\
\hline 11 & Clorpirifós $0,4 \%$ & 8 & 1,24 & 5,28 & 4,26 \\
\hline 12 & Clorpirifós $0,4 \%$ & 10 & 1,32 & 4,61 & 3,49 \\
\hline 13 & Testemunha & 0 & 0,96 & 4,22 & 4,40 \\
\hline
\end{tabular}

nigrosetosus apresentam maior número de olheiros por metro quadrado de ninho, quando esses são de menores tamanhos, pois formigueiros de $0,5 \mathrm{~m}^{2}$ apresentam 7,23 olheiros enquanto outros, com área viva acima de $3,5 \mathrm{~m}^{2}$ apresentam, em torno de 2,30 olheiros por metro quadrado. Formigueiros com área acima de dois metros quadrados apresentam número semelhante de olheiros (Fig. 1), o que pode indicar que à partir desse tamanho ocorre equilibrio entre a abertura de novos olheiros e o fechamento dos antigos. Finalmente, isto indica que a reposição de formigas, por novas, atinge equilibrio quando os formigueiros de $A$. laticeps nigrosetosus atingem cerca de dois metros quadrados, no qual esta espécie atinge a população máxima por ninho. Isto mostra, ainda, que esta espécie deve apresentar, apenas uma rainha por ninho, ao contrário de Acromyrmes subterraneus subterraneus
Forel (Hymenoptera: Formicidae), onde não é raro se encontrar um aumento no número de rainhas (Della Lucia \& Moreira, 1993). No entanto, espécies de saúvas, como Atta sexdens sexdens L. (Hymenoptera: Formicidae), apresentam redução do número de olheiros por metro quadrado com o aumento da área dos formigueiros, pois aqueles de $1 \mathrm{~m}^{2}$ e 100 $\mathrm{m}^{2}$ apresentam, respectivamente 0,5 e 0,1 olheiros $/ \mathrm{m}^{2}$ (Cruz et al., 1996). Além disso, Zanuncio et al. (1996a), relatam que o número de olheiros por metro quadrado, de formigueiro de Atta laevigata $\mathrm{F}$. Smith (Hymenoptera: Formicidae), diminui com o aumento da área do formigueiro, pois aqueles com área média de $0,35 \mathrm{~m}^{2}$ e de $13,99 \mathrm{~m}^{2}$ apresentam respectivamente, 6,48 e 0,81 olheiros por metro quadrado. Por outro lado, a diminuição do número de olheiros por metro quadrado, com o aumento da área de terra solta de förmigueiros de Atta 


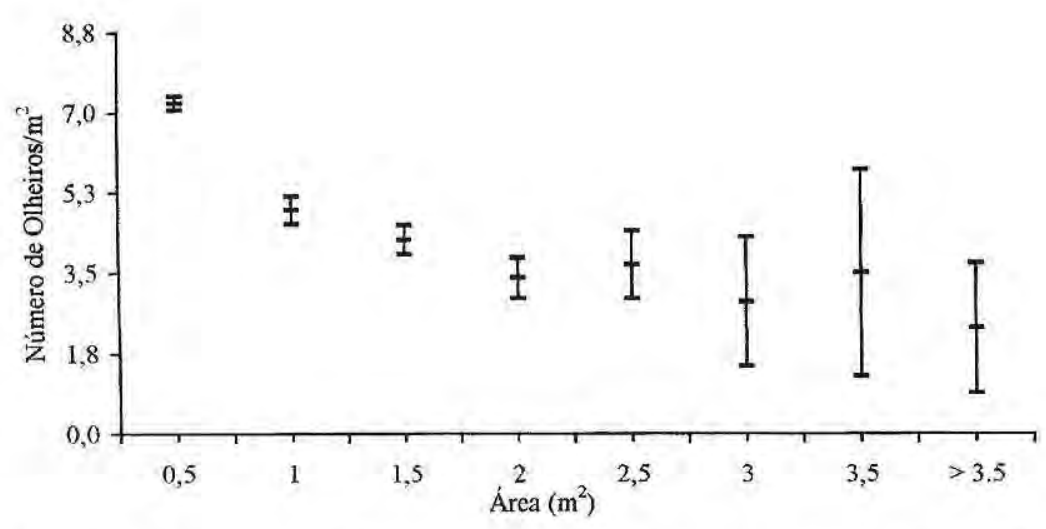

Figura 1. Relação entre área $\left(\mathrm{m}^{2}\right)$ e número de olheiros por metro quadrado de formigueiros de Acromyrmex laticeps nigrosetosus (Hymenoptera: Formicidae). Almerim, Pará.

talvez possa ser um padrão, já que espécies brasileiras desse gênero, apresentam apenas, uma rainha. Quando essa rainha atinge sua capacidade máxima de postura, ocorre, provavelmente, apenas reposição das operárias, que passam a atuar na construção de novas câmaras para a manutenção do fungo do qual se alimentam. Nesse caso, as câmaras antigas são abandonadas, constituindo áreas que, na superfície desses formigueiros, apresentam aparência de inativadas.

As duas iscas com sulfluramida apresentaram aceitação e eficiência semelhantes para $A$. laticeps nigrosetosus. $A$ isca com a sulfluramida $A$, a primeira a ser lançada no mercado nacional com esse ingrediente ativo, apresentou, para todas as dosagens, $100,00 \%$ de eficiência, com paralisação total, de todos os formigueiros tratados, aos 30 dias para as dosagens de 4, 6 e 10 gramas com, apenas, um formigueiro apresentando atividade, na avaliação final, na dosagem de 8 gramas por metro quadrado dessa isca. É interessante registrar a paralisação de
$61,11 \%$ dos formigueiros tratados, com essa isca, com 6 gramas por metro quadrado de formigueiro, aos três dias, enquanto as outras dosagens apresentaram, apenas $22,22 \%$ de formigueiros paralisados nessa data. A eficiência da isca com sulfluramida $\mathrm{A}$ foi semelhante aquela da isca com a sulfluramida B, que apresentou $100,00 \%$ de eficiência final, nas várias dosagens utilizadas. Zanuncio et al. (1996b) e Caetano et al. (1993), relatam alta eficiência da isca com a sulfluramida A, para o controle de Acromyrmex subterraneus molestans Santschi e de Acromyrmex aspersus F. Smith (Hymenoptera: Formicidae), respectivamente. Alta eficiência dessa isca, é relatada, também, para $A$. laevigata (Zanuncio et al. 1992), Atta bisphaerica Forel (Zanuncio et al. 1993), Atta cephalotes L. (Hymenoptera: Formicidae) (Zanuncio et al. 1996c) e para Atta sexdens rubropilosa Forel (Hymenoptera: Formicidae) (Zanuncio et al., 1997).

A isca à base de clorpirifós 
apresentou, também, boa aceitação por $A$. laticeps nigrosetosus e teve os melhores indices de paralisação de movimentação de formigas, no terceiro dia após sua aplicação. No entanto, essa isca apresentou menor eficiência $(55,56$ a $66,67 \%)$, o que concorda com os resultados de CRUZ et al. (1997), que relatam uma menor taxa de controle de $A$. sexdens sexdens, com essa isca, em comparação com aquelas à base de sulfluramida. Isto pode ser explicado pelo fato do clorpirifós ser um produto de contato, com efeito muito mais rápido que o da sulfluramida. No entanto, esse efeito muito rápido não é, normalmente, desejảvel em uma isca formicida, pois as formigas podem detectar o princípio ativo da mesma e paralisar sua incorporação ao fungo, o que reduzirá sua eficiência final.
Isto pode ser constatado, nas avaliações posteriores, pois a eficiência final da isca com clorpirifós foi de 55,56 a $66,67 \%$, nas dosagens de 4 a $10 \mathrm{~g} / \mathrm{m}^{2}$ de formigueiro.

Considerando que a eficiência aos 120 dias foi muito semelhante àquela registrada aos 30 dias (Tab. 2), recomenda-se a avaliação final dos ensaios de iscas com sulfluramida, para o controle de $A$. laticeps nigrosetosus, ao final de 30 dias, o que concorda com resultados relatados por Zanuncio et al. (1996b) para $A$. subterraneus molestans.

As iscas com sulfluramida são mais eficientes e portanto podem ser recomendadas para o controle de $A$. laticeps nigrosetosus, enquanto a isca à base de clorpirifós mostrou-se

Tabela 2. Porcentagem de aceitação das iscas e da paralisação e eficiência por tratamento e por dia de avaliação das iscas utilizadas para o controle de Acromyrmex laticeps nigrosetosus (Hymenoptera: Formicidae) em Almerim, Pará.

\begin{tabular}{|c|c|c|c|c|c|c|c|c|c|}
\hline \multirow{2}{*}{ Tratamento } & \multirow{2}{*}{ Isca Granulada } & \multirow{2}{*}{$\begin{array}{c}\text { Dosagem } \\
\left(\mathrm{g} / \mathrm{m}^{2}\right)\end{array}$} & \multicolumn{2}{|c|}{ Aceitaçāo $(\%)^{\text {aे }}$} & \multicolumn{4}{|c|}{ Paralisaçăo (\%) b } & \multirow{2}{*}{$\frac{\text { Eficiência (\%) }}{\text { Dia } 120}$} \\
\hline & & & 24 horas & 72 horas & Dia 3 & Dia 30 & Dia 50 & Dia 90 & \\
\hline 1 & Sulfluramida $A 0,3 \%$ & 4 & 99,89 & 99,89 & 22,22 & 100,00 & 100,00 & 100,00 & 100,00 \\
\hline 2 & Sulfluramida A 0,3\% & 6 & 100,00 & 100,00 & 61,11 & 100,00 & 100,00 & 100,00 & 100,00 \\
\hline 3 & Suffluramida A 0,3\% & 8 & 100,00 & 100,00 & 22,22 & 94,44 & 100,00 & 100,00 & 100,00 \\
\hline 4 & Suffluramida $A 0,3 \%$ & 10 & 99,61 & 100,00 & 22,22 & 100,00 & 100,00 & 100,00 & 100,00 \\
\hline 5 & Suffluramida B $0,3 \%$ & 4 & 99,44 & 100,00 & 5,56 & 94,44 & 94,44 & 94,44 & 100,00 \\
\hline 6 & Sufluramida B $0,3 \%$ & 6 & 99,94 & 100,00 & 5,56 & 100,00 & 100,00 & 100,00 & 100,00 \\
\hline 7 & Suffluramida B $0,3 \%$ & 8 & 100,00 & 100,00 & 16,67 & 100,00 & 100,00 & 100,00 & 100,00 \\
\hline 8 & Suffluramida B $0,3 \%$ & 10 & 99,67 & 100,00 & 27,78 & 100,00 & 100,00 & 100,00 & 100,00 \\
\hline 9 & Clorpiritós $0,4 \%$ & 4 & 98,89 & 99,89 & 50,00 & 77,78 & 66,67 & 77,78 & 61,11 \\
\hline 10 & Clorpiritós $0,4 \%$ & 6 & 100,00 & 100,00 & 27,78 & 77,78 & 72,22 & 72,22 & 66,67 \\
\hline 11 & Clorpiritós $0,4 \%$ & 8 & 93,89 & 100,00 & 33,33 & 66,67 & 66,67 & 66,67 & 66,67 \\
\hline 12 & Clorpiritós $0,4 \%$ & 10 & 98,94 & 100,00 & 50,00 & 77,78 & 72,22 & 66,67 & 55,56 \\
\hline 13 & Testemunha & 0 & 100,00 & 100,00 & 0,00 & 5,56 & 0,00 & 0,00 & 0,00 \\
\hline
\end{tabular}

a) \% das iscas carregadas; $\left.{ }^{b}\right) \%$ dos ninhos que nẫo estavam em atividade; c) \% de ninhos mortos 
menos eficiente e por isto não deve ser recomendada para este fim.

\section{CONCLUSÕES}

As iscas à base de sulfluramida, apresentaram $100,00 \%$ de eficiência, enquanto aquela à base de clorpirifós apresentou de 55,56 a $66,67 \%$ de eficiência. Devido ao seu maior efeito de contato, esta isca apresentou, aos três dias, maior taxa de paralisação de formigueiros de $A$. laticeps nigrosetosus que aquelas à base de sulfluramida. Embora a avaliação tenha sido feita até os 120 dias, esta pode ser feita até aos 30 dias, para as iscas com sulfluramida, pois nas demais avaliações, os resultados foram semelhantes. No entanto, isto não deve ser feito para a isca com clorpirifós, pois muitos dos formigueiros tratados com a mesma, apresentaram aumento da atividade de forrageamento a partir dos 30 dias de avaliação.

\section{AGRADECIMENTOS}

À CAPES, CNPq e FAPEMIG pelas bolsas e auxílios concedidos. A Jari Celulose S.A., pelo desenvolvimento desta pesquisa em suas áreas.

\section{Bibliografia citada}

Amante, E. 1972. Preliminary observations on the swarming behaviour of the leaf-cutting ant, Atta capiguara (Hymenoptera: Formicidae). Journal of the Georgia Entomological Society, 7: 82-83.

Caetano, F.H.; Pacheco, P.; Costa-Coelho, L.C. 1993. Verificação da ação do sulfluramidGX-071 HB, isca granulada formicida sobre colônias de Acromyrmex aspersus
(Hymenoptera: Formicídae). In: Anais do $14^{\circ}$ Congresso Brasileiro de Entomologia, Sociedade Entomológica do Brasil, Piracicaba, São Paulo, p. 517.

Cherrett, J.M. 1968. The foraging bahaviour of Atta cephalotes (L.) (Hymenoptera: Formicidae). I. Foraging pattern and plant species attacked in tropical rain forest. Journal of Animal Ecology, 37: 387-402.

Cruz, A.P.; Zanuncio, J.C.; Zanetti, R.; Gomes, O. S. 1996. Eficiência de iscas granuladas à base de sulfluramida e de clorpirifós no controle de Atta sexdens sexdens (Hymenoptera: Formicidae), no trópico úmido. Acta Amazonica, 26: 145-150.

Cruz, A.P.; Zanuncio, J.C.; Zanetti, R.; Pereira, J.M.M. 1997. Eficiência de iscas formicidas à base de sulfluramida e clorpirifós no controle de Acromyrmex octospinosus (Hymenoptera: Formicidac). In: Resumos do $16^{\circ}$ Congresso Brasileiro de Entomologia. Salvador, BA, p. 252.

Della Lucia, T.M.C.; Fowler, H.G.;Moreira, D.D.O. 1993. Espécies de formigas cortadeiras no Brasil. In: Della Lucia, T.M.C. (ed.). As Formigas Cortadeiras. Viçosa. p. 26-31.

Della Lucia, T.M.C.; Moreira, D.D.O. 1993. Caracterização dos ninhos. In: Della Lucia, T.M.C. (ed.). As Formigas Cortadeiras. Viçosa. P. 26-31.

Gonçalves, C.R. 1961. O gênero Acromyrmex no Brasil. Studia Ent., 4: 113-180.

Mariconi, F.A.M. 1970. As Saúvas. São Paulo, Agronômica, Ceres. 167p.

Vilela, E.F. 1986. Status of leaf-cutting ant control in forest plantations in Brazil. In: Lofgreen, C.S.; Vander Meer, R.K. (eds.) Fire ants and leaf-cutting ants: biology and management. Boulder, Westview Press. p. 399-408.

Zanuncio, J.C.; Couto, L.; Santos, G.P.; Zanuncio, T.V. 1992. Eficiência da isca granulada à base de sulfluramid, no controle da formiga cortadeira Atta laevigata (F. Smith, 1858) (Hymenoptera: Formicidae). Revista Arvore, 17: 85-90.

Zanuncio, J.C.; Couto, L.; Fagundes, M.; 
Zanuncio, T.V. 1993. Eficiência da isca Mirex-S, (sulfluramid 0,3\%) no controle da formiga cortadeira Atta bisphaerica Forel (Hymenoptera: Formicidae). Revista Arvore, 17: 85-90.

Zanuncio, J.C.; Torres, J.B.; Gasperazzo, W.L.; Zanuncio, T.V. 1996a. Aferição de dosagens de iscas granuladas para o controle de Atta laevigata (F. Smith) pelo número de olheiros ativos. Revista Arvore, 20: 241-246.

Zanuncio, J.C.; Laranjeiro, A.J.; Souza de, O.F. 1996b. Controle de Acromyrmex subterraneus molestans Santschi (Hymenoptera: Formicidae) com sulfluramida. Anais da Sociedade Entomológica do Brasil, 25: 383-388.

Zanuncio, J.C; Cruz, A.P.; Santos, G.P.; Oliveira, M.A. 1996c. Eficiência da isca Mirex-S (sulfluramida $0,3 \%$ ), em três dosagens, no controle de Atta cephalotes (Hymenoptera: Formicidae). Acta Amazonica, 26: 115-120.

Zanuncio, J.C.; Santos, G.P.; Firme, D.J.; Zanuncio, T.V. 1997. Uso da isca granulada com sulfluramid 0,3\% no controle de Atta sexdens rubropilosa Forel, 1908 (Hymenoptera: Formicidae). Cerne, 3: 47-54. 